\title{
Comparison of Position Estimation Techniques Using Occupancy Grids
}

\author{
Bernt Schiele and James L. Crowley \\ LIFIA-INPG \\ 46 Avenue Félix Viallet \\ 38031 Grenoble Cedex \\ FRANCE \\ schiele@imag.fr jlc@imag.fr \\ Phone: (33) 76574655
}

\begin{abstract}
This paper addresses the problem of perception and localisation for a mobile robot in an unknown environment. It describes a modelling of the environment based on two certainty grids: one local model and the other one a global model. Localisation of the robot is reduced to finding the best alignment of the local certainty grid onto the global certainty grid. Four localisation procedures to correct the robot position are introduced. First experimental results show the capacity of the method.
\end{abstract}

\section{Introduction}

Certainty Grids have been proposed as a way to construct an internal model of static environments based on sensor data ([Mor 85, Mor 88]). This method takes into account the uncertainty of sensory data by working with probabilities or certainty values. The certainty grid representation can be used directly in robotic planning ([Wal 92]) or navigation ([Elf 89]). Other authors have used a certainty grid method for collision avoidance ([Bor 91, Bor 90]). Two drawbacks of the certainty grid method have not been solved so far satisfactorily. One is the modelling of dynamic obstacles and the second is the localisation process for the robot.

In this paper we offer a solution for the localisation problem in mobile robotics. We use a modelling of the environment which is based on two certainty grids. The first certainty grid is centered on the robot and models its vicinity. The second certainty grid is the global model of the environment where we do not suppose an a-priori model. The goal of the paper is to introduce and compare localisation procedures based on these two certainty grids that have been implemented in our robot.

Section 2 introduces the modelling of the environment using two certainty grids. The two following sections 3 and 4 describe the re-localisation procedures we have implemented in our robot. Section 3 describes the matching procedures for the re-localisation procedures and section 4 the Kalman filters we are using for the updating of the estimated robot position. Section 5 introduces the integration of the local certainty grid data into the global certainty grid. In section 6 the first experimental results of the described localisation procedures are discussed. A more detailed description of the perception and localisation process is given in [Sch 93].

\section{Certainty Grids}

The certainty grid method for modelling of a mobile robot environment is mainly motivated to obtain a map of free space, in order to compute safe trajectories for the vehicle. This modelling is suitable for planning of safe trajectories, navigation and collision avoidance for a mobile robot.

In the certainty grid method ([Mor 85]) the space is represented by a regular grid with each cell holding a certainty value that a particular patch of space is occupied. The certainty value is based only on sensor readings. This section introduces a model of the robot environment based on two certainty grids. The first is centered on the robot and models the vicinity of the robot. The second has global parameters and models the entire environment. We do not suppose to have an a-priori model of the environment. Rather the robot "explores" the environment in order to build the global model. 
The local certainty grid contains information about the environment of the robot which is directly perceivable. Therefore, the grid is centered on the robot and the robot integrates the sensory data into the local grid. This local certainty grid is suitable to avoid collisions with static and with dynamic obstacles. As described in 3 and 4, the local certainty grid is used together with the global certainty grid for the correction of the estimated robot position.

In order to avoid collisions with static and dynamic obstacles the updating process of the local certainty grid has two important characteristics: On the one hand, the updating process is rapid so that we can update frequently. And on the other hand, the data of the local grid becomes outdated. Consequently, the local certainty grid always contains the most present data of the vicinity of the robot.

The resolution and the region of this grid depend on the velocity of the robot. When the robot is moving relatively fast, the region of the local grid grows at expense of the resolution. It is obvious that we do not obtain the best correction of the estimated robot position with a poor resolution. But at the moment of high robot velocity it is much more important to avoid collisions with static and dynamic obstacles as it is possible with the enlarged region of the local grid. The opposite is true for a low vehicle velocity where we have a smaller region and a better resolution of the local grid. With such a local grid we can correct the estimated position more accurately. Lower velocities are typical in the vicinity of the goal position so that the robot can reach this position accurately.

\subsection{Global Certainty Grid}

The global certainty grid is the two-dimensional representation of the static environment. The initial state of the global certainty grid is completely unknown because we do not provide an a-priori model of the environment. While moving, the robot explores the environment by integrating the observations into the global grid. As described in 2.1 the robot integrates the observations into the local certainty grid that models the vicinity of the robot. The updating process of the global certainty grid integrates the data of the local certainty grid into the global certainty grid (see 5).

The global certainty grid can be used for global path-planning and together with the local grid to correct the estimated position of the robot (see 3 and 4). In the future, it should be possible to also model dynamic objects in this global certainty grid. Zhang and Webber ([Zha 92]) have proposed a modified Hough-transform to detect moving objects. However the described method currently works only with a few integer velocities and is relatively time consuming.

\subsection{Finding Line Segments in the Grids}

A characteristic of man made environments is that objects tend to lie in straight lines. Examples are walls and doorways. It is possible to use line segments for the correction of the estimated position as explained in section 3 . Such straight lines can be found in the certainty grids as aligned cells of high probability of occupation. By interpreting a grid and its probabilities as an image with different levels of resolution we can apply vision functions for the search of straight lines.

Our current method for searching straight lines is an extended Hough-transform. The ordinary Houghtransform ([Bal 82]) is based on a function shown in equation (1). In this function, $(x, y)$ are the coordinates of a point and $(\rho, \theta)$ are the parameters of a straight line. As illustrated in figure 1, $\rho$ is the perpendicular distance of the straight line to the origin and $\theta$ is the angle of the normal with the $\mathrm{x}$-axis. The function (1) computes for a certain $(\rho, \theta)$-couple all points $((x, y)$-couples $)$ which lie on the same straight line. But this function computes as well for a certain point $((x, y)$-couple $)$ all the straight lines $((\rho, \theta)$-couples $)$ passing that point. The Hough-transform enters for a certain point (possible member of a straight line) all straight lines passing that point into a Hough-parameterspace (which is indexed with $\rho$ and $\theta$ ). Such a certain point is in our case a grid-cell with a high probability to be occupied. Local maxima of the Hough-space represent straight lines which exist in the grid.

$$
f(x, y, \rho, \theta)=x \cos \theta+y \sin \theta-\rho=0
$$

Our extension of the Hough-transform is to compute a probability for a $(\rho, \theta)$-couple and to compute the uncertainty $\left(\sigma_{\rho}^{2}, \sigma_{\theta}^{2}\right)$ of that couple. For a $(\rho, \theta)$-couple representing a infinite line, we find the beginning and ending point of the line segment lying in our certainty grid. To arrive at the probability, we look at the probabilities of the grid-cells lying on the line segment and in a certain region around the line segment. The computation of the uncertainty $\left(\sigma_{\rho}^{2}, \sigma_{\theta}^{2}\right)$ of a $(\rho, \theta)$-couple is based on the Hough-space. As described above, the $(\rho, \theta)$-couple of a line segment is a local maxima in the Hough-space. If we model the local maxima in 
Our segment representation is composed of the following parameters (see figure 1):

- $\rho$ : perpendicular distance of the segment to the origin with uncertainty $\sigma_{\rho}^{2}$,

$-\theta$ : angle of the normal with the $\mathrm{x}$-axis with uncertainty $\sigma_{\theta}^{2}$,

- $(x, y)$ : coordinates of the midpoint and

$-h$ : half length of the segment.

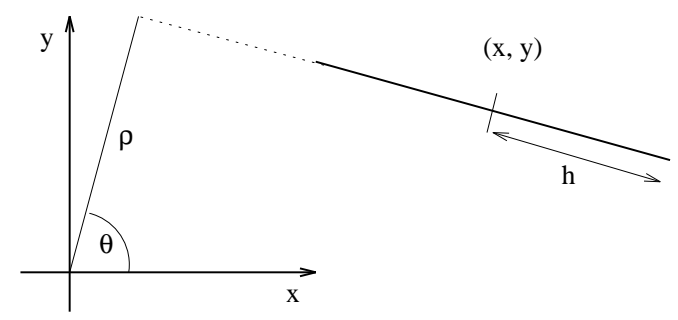

Fig. 1.: Representation of a line segment

\section{Matching Certainty Grids}

In this section four matching procedures for the re-localisation process of the robot are introduced. Because these procedures are based on an estimated position of the robot we call this process "correction of the estimated position". The estimated position of the robot $\hat{r}=\left(\hat{r}_{x} \hat{r}_{y} \hat{r}_{\alpha}\right)^{T}$ and the covariance of that estimation $C_{r}$ is given by wheel-encoders of the robot.

As described in 2.3 we can find line segments in the grids with a Hough-transform. Thus, the first relocalisation process works with segments obtained of the local segments and from the global segment. This process is composed of three parts:

1. Finding line segments in the two grids with our extended Hough-transform,

2. matching the segments (see 3.1 ) and

3. applying two independent Kalman filters for the correction of the orientation $r_{\alpha}$ and the position $\left(r_{x} r_{y}\right)^{T}$ of the robot (see 4.1 and [Cro 89]).

The other re-localisation processes search the best transformation $t=\left(t_{x} t_{y} t_{\alpha}\right)^{T}$ of the local grid into the global grid. Three matching procedures are described to find the best transformation $t$ and its covariance $C_{t}$ :

- Matching local segments directly against the global certainty grid (see 3.2),

- matching global segments directly against the local certainty grid (see 3.3 ) and

- matching directly the two certainty grids (see 3.4 ).

After we have found such a transformation $t$ and its covariance $C_{t}$, we also apply a Kalman filter to correct the robot position (see 4.2 ).

The matching procedures described in this section allow to decrease the uncertainty of the robot position with the help of Kalman filters (see 4). In consideration of this uncertainty we integrate the data of the local grid into the global grid (see 5) in order to obtain a global model of the environment.

\subsection{Matching Local Segments against Global Segments}

The first re-localisation process starts with finding segments in the two certainty grids. As segments are obtained with the extended Hough-transform from the local grid (see 2.3) they are matched against segments obtained from the global grid.

Two segments $S_{l o}$ and $S_{g l}$ are given, one of the local and the other one of the global grid with the following parameters (see figure 1 ):

$$
\begin{aligned}
& S_{l o}:\left(\theta_{l o}, \sigma_{\theta_{l o}}^{2}, \rho_{l o}, \sigma_{\rho_{l o}}^{2}, x_{l o}, y_{l o}, h_{l o}\right) \\
& S_{g l}:\left(\theta_{g l}, \sigma_{\theta_{g l}}^{2}, \rho_{g l}, \sigma_{\rho_{g l}}^{2}, x_{g l}, y_{g l}, h_{g l}\right)
\end{aligned}
$$




$$
\begin{gathered}
\left(\theta_{g l}-\theta_{l o}\right) \leq \sigma_{\theta_{l o}}^{2}+\sigma_{\theta_{g l}}^{2} \\
\left(\rho_{g l}-\rho_{l o}\right) \leq \sigma_{\theta_{g l}}^{2}+\sigma_{\rho_{g l}}^{2} \\
\left(x_{g l}-x_{l o}\right)^{2}+\left(y_{g l}-y_{l o}\right)^{2} \leq h_{g l}+h_{l o}
\end{gathered}
$$

To the segment with the highest probability that has passed the these three comparison test we apply two independent Kalman filters. These filters are described in 4.1.

\subsection{Matching Local Segments against the Global Grid}

In the second re-localisation process the global grid is matched with local segments obtained from the local certainty grid. Therefore, the process starts with finding line segments in the local certainty grid, continues by computing the best transformation of the segments into the global grid and ends by applying the Kalman filter which is described in 4.2 .

For matching a local segment directly against the global grid, we produce a mask of the segment. This mask contains the probabilities of the local grid cells lying on this line segment. This mask is transformed into the global certainty grid and correlated with the global grid cells lying under this mask. The value of that correlation increases when the cells are of the same state (both have a probability to be occupied or both have a probability to be free). On the other hand the value decreases when two cells have different states (one cell has a probability to be occupied and the other to be free). The value of that correlation is the basis for the estimation of the quality of the transformation. By varying the transformation of the segment mask into the global grid we find the best transformation between the two grids. With the estimated position of the robot we have also an estimation of the transformation between the two grids which we are using to find the best transformation.

The computation of the covariance $C_{t}$ is based of the values of the correlations around the best transformation. The distribution of the correlation values are supposed to be of Gaussian form. So we can compute the covariance $C_{t}$ of the transformation $t$ by analysing the distribution of the correlation values.

\subsection{Matching the Local Grid against Global Segments}

The principle of the third re-localisation process is the same as of the second re-localisation process (see 3.2). The sole difference is that we are using global segments and directly the local certainty grid. If we have found the best transformation of a global segment into the local grid, we apply as well the Kalman filter described in 4.2 .

\subsection{Matching the Local Grid against the Global Grid}

The fourth re-localisation process also searches for the transformation of the local grid into the global grid. This time we directly use the two certainty grids. It is possible to correlate the entire local grid with the global grid. But we have decided to use only a part of the local certainty grid and to find the best transformation of this part into the global certainty grid. One reason for that selection is the data reduction. But as well the free regions of the environment are not suitable for the re-localisation process. So we produce a mask of the local grid, which contains the grid cells with a probability to be occupied above a certain threshold. This mask is used in the same manner as the segment masks to find the best transformation between the two grids (see 3.2). After finding the best transformation $t$ and the covariance $C_{t}$ of this transformation we apply the Kalman filter described in 4.2 .

\section{Updating the Estimated Position of the Robot}

Because the parameters of the global grid are absolute and those of the local grid are relative to the robot the uncertainty of the local grid relatively to the global grid is the same as the uncertainty of the robot position $r=\left(r_{x} r_{y} r_{\alpha}\right)^{T}$. If we can correct the position of the local grid, we can correct the robot position. The following section 4.1 presents the Kalman filters for the first re-localisation process which is based on segments obtained from the two certainty grids (see 3.1). The section 4.2 discusses the Kalman filter for the other re-localisation processes (see 3.2, 3.3 and 3.4).

For the updating of the estimated position of the robot we use a Kalman filter ([Bab 78, Cro 89, Cro 92]). Here we want introduce only the notions of the Kalman filter for the linear and discrete case that will be used in the following: 
$\Phi_{k / k-1}$ is the transition matrix between the state $X_{k-1}$ and $X_{k} . W_{k}$ is the noise of the model with expectation zero and known covariance $Q_{k}$.

$-Z_{k}$ is the observation vector at the instant $k$. The connection of the observation and the state vector is given by:

$$
Z_{k}=H_{k} X_{k}+V_{k}
$$

$H_{k}$ is the observation matrix. $V_{k}$ is the noise with expectation zero and known covariance $R_{k}$.

The prediction (or the estimation) of the state vector $\hat{X}_{k+1 / k}$ (for the following instant $k+1$ ), the error of this prediction $\hat{P}_{k+1 / k}$ and the prediction of the observation vector $\hat{Z}_{k+1 / k}$ are given by:

$$
\begin{aligned}
\hat{X}_{k+1 / k} & =\Phi_{k+1 / k} \hat{X}_{k} \\
\hat{P}_{k+1 / k} & =\Phi_{k+1 / k} \hat{P}_{k} \Phi_{k+1 / k}^{T}+Q_{k} \\
\hat{Z}_{k+1 / k} & =H_{k+1} \hat{X}_{k+1 / k}
\end{aligned}
$$

In the equations of the Kalman filter we compute an intermediary $K_{k+1}$ called Kalman gain for the correction of the predicted state vector $\hat{X}_{k+1}$ and of the predicted error $\hat{P}_{k+1}$ :

$$
\begin{aligned}
K_{k+1} & =\hat{P}_{k+1 / k} H_{k+1}^{T}\left(H_{k+1} \hat{P}_{k+1 / k} H_{k+1}^{T}+R_{k+1}\right)^{-1} \\
\hat{X}_{k+1} & =\hat{X}_{k+1 / k}+K_{k+1}\left(Z_{k+1}-\hat{Z}_{k+1 / k}\right) \\
\hat{P}_{k+1} & =\hat{P}_{k+1 / k}-K_{k+1} H_{k+1} \hat{P}_{k+1 / k}
\end{aligned}
$$

\subsection{Kalman Filters for the First Re-localisation Process}

The first re-localisation process is based on local and global segments obtained from the two certainty grids. Each match of a local segment with a global segment (see 3.1) provides a one dimensional constraint on the position of the robot $\left(r_{x} r_{y}\right)^{T}$ and its uncertainty as well as a constraint on the orientation of the robot $r_{\alpha}$. These constraints may be applied using two Kalman filters ([Cro 89]).

The first Kalman filter corrects the robot orientation $r_{\alpha}$ and using consequently the robot orientation as the state vector (8). The filter uses the difference of the orientations of the matched segments as observation vector. In the ideal case this difference computes as well the robot orientation (9). The observation "matrix" is than given by (10) and the noise of the observation by the covariance of the obtained segments (11).

The prediction of the robot orientation $\hat{X}_{k+1 / k}$ (12) and the error of this prediction $\hat{P}_{k+1 / k}(13)$ is given by the robot. The prediction of the observation $\hat{Z}_{k+1 / k}$ is then the predicted orientation of the robot (13).

$$
\begin{aligned}
X_{k} & =r_{\alpha} \\
Z_{k} & =\theta_{g l}-\theta_{l o}=r_{\alpha} \\
H_{k} & =1 \\
R_{k} & =\sigma_{\theta_{g l}}^{2}+\sigma_{\theta_{l o}}^{2}
\end{aligned}
$$

$$
\begin{aligned}
& \hat{X}_{k+1 / k}=\hat{r}_{\alpha} \\
& \hat{Z}_{k+1 / k}=\hat{r}_{\alpha} \\
& \hat{P}_{k+1 / k}=\sigma_{\alpha}^{2}
\end{aligned}
$$

After the equations of the Kalman filter (see equations (5), (6) and (7)) we can correct the predicted robot orientation $\hat{X}_{k+1}$ and the precision of this correction $\hat{P}_{k+1}$ by the following equations:

$$
\begin{aligned}
& K_{k+1}=\frac{\sigma_{\alpha}^{2}}{\sigma_{\theta_{g l}}^{2}+\sigma_{\theta_{l o}}^{2}} \\
& \hat{X}_{k+1}=\hat{r}_{\alpha}+\frac{\sigma_{\alpha}^{2}}{\sigma_{\theta_{g l}}^{2}+\sigma_{\theta_{l o}}^{2}}\left(\theta_{g l}-\theta_{l o}-\hat{r}_{\alpha}\right) \\
& \hat{P}_{k+1}=\sigma_{\alpha}^{2}-\frac{\sigma_{\alpha}^{2}}{\sigma_{\theta_{g l}}^{2}+\sigma_{\theta_{l o}}^{2}} \sigma_{\alpha}^{2}
\end{aligned}
$$

The second Kalman filter corrects the robot position $\left(r_{x} r_{y}\right)^{T}$. Therefore, we use the vector $\left(r_{x} r_{y}\right)^{T}$ as the state vector (18). We apply the correction of the robot position only in the direction perpendicular to the segment. Consequently we use the perpendicular distance of the local segment to the robot as observation 
The predictions for the state vector $\hat{X}_{k+1 / k}(22)$ and the error of this prediction $\hat{P}_{k+1 / k}(24)$ are estimated by the robot. The predicted observation $\hat{Z}_{k+1 / k}$ is then given by (23).

$$
\begin{aligned}
& X_{k}=\left(r_{x} r_{y}\right)^{T} \\
& Z_{k}=\rho_{l o} \\
& H_{k}=\left(\begin{array}{ll}
\sin r_{\alpha} & -\cos r_{\alpha}
\end{array}\right) \\
& R_{k}=\sigma_{\rho_{g l}}^{2}+\sigma_{\rho_{l o}}^{2}
\end{aligned}
$$

$$
\begin{aligned}
\hat{X}_{k+1 / k} & =\left(\hat{r}_{x} \hat{r}_{y}\right)^{T} \\
\hat{Z}_{k+1 / k} & =\hat{r}_{x} \sin r_{\alpha}-\hat{r}_{y} \cos r_{\alpha} \\
\hat{P}_{k+1 / k} & =\left(\begin{array}{cc}
\sigma_{x}^{2} & \sigma_{x y} \\
\sigma_{y x} & \sigma_{y}^{2}
\end{array}\right)
\end{aligned}
$$

After the equations of the Kalman filter (see equations (5), (6) and (7)) we can correct the predicted robot position $\hat{X}_{k+1}$ and the precision of this correction $\hat{P}_{k+1}$ by the following equations:

$$
\begin{aligned}
K_{k+1} & =\left(\begin{array}{l}
k_{1} \\
k_{2}
\end{array}\right)=\frac{1}{\sigma_{\rho_{g l}}^{2}+\sigma_{\rho_{l}}^{2}}\left(\begin{array}{c}
\sigma_{x}^{2} \sin r_{\alpha}-\sigma_{x y} \cos r_{\alpha} \\
\sigma_{y x} \sin r_{\alpha}-\sigma_{y}^{2} \cos r_{\alpha}
\end{array}\right) \\
\hat{X}_{k+1} & =\left(\begin{array}{ll}
\hat{r}_{x}+k_{1}\left(\rho_{l o}-\hat{r}_{x} \sin r_{\alpha}+\hat{r}_{y} \cos r_{\alpha}\right) \\
\hat{r}_{y}+k_{2}\left(\rho_{l o}-\hat{r}_{x} \sin r_{\alpha}+\hat{r}_{y} \cos r_{\alpha}\right)
\end{array}\right) \\
\hat{P}_{k+1} & =\hat{P}_{k+1 / k}-\left(\begin{array}{ll}
k_{1} \sin r_{\alpha} & -k_{1} \cos r_{\alpha} \\
k_{2} \sin r_{\alpha} & -k_{2} \cos r_{\alpha}
\end{array}\right) \hat{P}_{k+1 / k}
\end{aligned}
$$

\subsection{Kalman for the second, third and fourth Re-localisation Process}

In $3.2,3.3$ and 3.4 we have introduce matching procedures which find the best transformation $t$ of the local grid into the global grid. In this section we discuss the Kalman filter which is based on this transformation $t$ and which will correct the robot position $\left(r_{x} r_{y} r_{\alpha}\right)^{T}$.

As state vector $X_{k}$ of this Kalman filter we use the whole robot position (28). As observation vector we can apply directly the transformation $t$ (29). In the ideal case this transformation $t$ of the local grid into the global grid is equivalent to the robot position. Therefore, the observation matrix is the unity matrix (30). In 3.2 we have explained the computation of the covariance matrix $C_{t}$ which is also the covariance of the observation. The matrix is given in (31).

The prediction of the state vector $\hat{X}_{k+1 / k}(32)$ as well the prediction of the observation vector $\hat{Z}_{k+1 / k}$ (33) is given by the robot. Also the covariance of the predicted state vector $\hat{P}_{k+1 / k}$ is given by the robot (34).

$$
\begin{aligned}
& X_{k}=r=\left(r_{x} r_{y} r_{\alpha}\right)^{T} \\
& Z_{k}=t=\left(t_{x} t_{y} t_{\alpha}\right)^{T} \\
& H_{k}=I \\
& R_{k}=C_{t}=\left(\begin{array}{ccc}
\sigma_{t_{x}}^{2} & \sigma_{t_{x y}} & \sigma_{t_{x \alpha}} \\
\sigma_{t_{y x}} & \sigma_{t_{y}}^{2} & \sigma_{t_{y \alpha}} \\
\sigma_{t_{\alpha x}} & \sigma_{t_{\alpha y}} & \sigma_{t_{\alpha}}^{2}
\end{array}\right)
\end{aligned}
$$

$$
\begin{aligned}
& \hat{X}_{k+1 / k}=\hat{r}=\left(\hat{r}_{x} \hat{r}_{y} \hat{r}_{\alpha}\right)^{T} \\
& \hat{Z}_{k+1 / k}=\hat{r}=\left(\hat{r}_{x} \hat{r}_{y} \hat{r}_{\alpha}\right)^{T} \\
& \hat{P}_{k+1 / k}=C_{r}=\left(\begin{array}{ccc}
\sigma_{r_{x}}^{2} & \sigma_{r_{x y}} & \sigma_{r_{x \alpha}} \\
\sigma_{r_{y x}} & \sigma_{r_{y}}^{2} & \sigma_{r_{y \alpha}} \\
\sigma_{r_{\alpha x}} & \sigma_{r_{\alpha y}} & \sigma_{r_{\alpha}}^{2}
\end{array}\right)
\end{aligned}
$$

After the equation of the Kalman filter (see equations (5), (6) and (7)) we compute the Kalman gain $K_{k+1}$ and the correction of the predicted state vector $\hat{X}_{k+1}$ and his predicted covariance $\hat{P}_{k+1}$ :

$$
\begin{aligned}
K_{k+1} & =C_{r}\left(C_{r}+C_{t}\right)^{-1} \\
\hat{X}_{k+1} & =\hat{r}+C_{r}\left(C_{r}+C_{t}\right)^{-1}(t-\hat{r}) \\
\hat{P}_{k+1} & =C_{r}-C_{r}\left(C_{r}+C_{t}\right)^{-1} C_{r}
\end{aligned}
$$

\section{$5 \quad$ Updating the Global Grid}

The updating process of the global certainty grid integrates the data of the local certainty grid into the global grid. It is indispensable, that the updating process takes into account the uncertainty of the local grid position. Because the local grid is centered on the robot, this uncertainty is the uncertainty of the robot position. We remark that the correction of the estimated position of the robot is very important for the updating process particular during exploration of unknown environment. 


$$
\operatorname{global-grid}(x, y)= \begin{cases}\text { conv-grid }(x, y) & \text { if global-grid }(x, y)=\text { unknown } \\ \frac{1}{2}(\text { conv-grid }(x, y)+\operatorname{global}-\operatorname{grid}(x, y)) \text { otherwise }\end{cases}
$$

In this formula global-grid $(x, y)$ is the global certainty grid with the absolute parameters $x$ and $y$. conv$\operatorname{grid}(x, y)$ is the local grid convoluted with the uncertainty of the robot position and transformed to the absolute parameters.

\section{Experimental Results}

We have incorporated the perception and the re-localisation procedures described in this paper into the overall system of our mobile robot platform. After the first series of experiments we found that specially the first and the fourth re-localisation procedures generate stable results for the re-localisation.

The first re-localisation procedure uses segments retrieved from the local grid and from the global grid (see 3.1 and 4.1). The results are comparable or more accurate than the ones we obtain with our previous approach which is based on a parametric model. This latter extracts directly line segments from the sensory data $([$ Cro 89$])$.

The fourth re-localisation procedure correlates a part of the local certainty grid with the global certainty grid (see 3.4 and 4.2). The covariance we obtain from such a correlation is relatively large. However, by each application of the re-localisation procedure we can make an accurate correction of the estimated robot position. Thereby, repeated application reduces the uncertainty of the robot position. Because of the reduced data the procedure is computed in real time.

\section{Conclusion}

In this paper we have considered a certainty grid method for perception and localisation in order to model the robot environment and to correct the robot position with this representation of the environment. Therefore, we have introduced a local certainty grid which is centered on the robot and models the vicinity of the robot. A second certainty grid, the global grid contains the two-dimensional representation of the environment. We have described four localisation processes using a Kalman filter to reduce the uncertainty of the estimated robot position. First experimental results show the capacity of the method.

\section{References}

[Bab 78] M. Babarrere, P. P. Krief, and B. Gimonet. Le Filtrage et ses Applications. Cepaclus Edition, 1978.

[Bal 82] D. H. Ballard and C. M. Brown. Computer Vision. Englewood Cliffs, NJ: Prentice Hall, 1982.

[Bor 90] J. Borenstein and Y. Koren. Real-time obstacle avoidance for fast mobile robots in cluttered environments. In IEEE International Conference on Robotics and Automation, pages 572-577, 1990.

[Bor 91] J. Borenstein and Y. Koren. The vector field histogram - fast obstacle avoidance for mobile robots. IEEE Transactions on Robotics and Automation, 7(3): 278-288, June 1991.

[Cro 89] J. L. Crowley. World modelling and position estimation for a mobile robot using ultrasonic ranging. In IEEE International Conference on Robotics and Automation, pages 674-680, 1989.

[Cro 92] J. L. Crowley, P. Stelmaszyk, T. Skordas, and P. Puget. Measurement and integration of 3-d structures by tracking edge lines. International Journal of Computer Vision, 8(1): 29-52, 1992.

[Elf 89] A. Elfes. Using occupancy grids for mobile robot perception and navigation. IEEE Computer, pages 46-57, Jume 1989.

[Mor 85] H. P. Moravec and A. Elfes. High resolution maps from angle sonar. In IEEE International Conference on Robotics and Automation, pages 116-121, March 1985.

[Mor 88] H. P. Moravec. Sensor fusion in certainty grids for mobile robots. AI Magazine, 9(2): 61-74, 1988.

[Sch 93] B. Schiele. Perception et Localisation pour un Robot Mobile. Technical report, DEA Informatique, LIFIA/INP Grenoble, 1993.

[Wal 92] F. Wallner, T. C. Lueth, and F. Langinieux. Fast local path planning for a mobile robot. In ICARCV'92 Second International Conference on Automation, Robotics and Computer Vision, September 1992.

[Zha 92] Y. Zhang and R. E. Webber. On combining the hough transform and occupancy grid methods for detection of moving objects. In IEEE/RSJ International Conference on Intelligent Robots and Systems, pages 21552160, July 1992 .

This article was processed using the TEX macro package with IRS93 style 